\title{
A Study on Synergistic Development of All-for-One Ecotourism of Chengdu-Chongqing Urban Cluster
}

\author{
Zibiao Cheng1, Chengduan Wang ${ }^{2}$ \\ ${ }^{1}$ College of Financial and Economic Management, Sichuan University of Arts and Science, Dazhou, China \\ ${ }^{2}$ Party Committee Office, Sichuan University of Arts and Science, Dazhou, China \\ Email: 47872614@qq.com
}

How to cite this paper: Cheng, Z.B. and Wang, C.D. (2019) A Study on Synergistic Development of All-for-One Ecotourism of Chengdu-Chongqing Urban Cluster. Open Access Library Journal, 6: e5379. https://doi.org/10.4236/oalib.1105379

Received: April 9, 2019

Accepted: May 5, 2019

Published: May 8, 2019

Copyright $\odot 2019$ by author(s) and Open Access Library Inc.

This work is licensed under the Creative Commons Attribution International License (CC BY 4.0).

http://creativecommons.org/licenses/by/4.0/ (c) (†) Open Access

\begin{abstract}
Chengdu-Chongqing Urban Cluster boasts abundant cultural and natural tourism resources. However, its socioeconomic effect remains non-prominent due to backward infrastructure, shortage of ecotourism talents and insufficient synergistic development. We must seek to promote a synergistic development of all-for-one ecotourism so as to enhance the socioeconomic effect of Chengdu-Chongqing Urban Cluster. All these cannot do without concerted efforts from government, tourism businesses and tourists.
\end{abstract}

\section{Subject Areas}

Socioeconomics

\section{Keywords}

Chengdu-Chongqing Urban Cluster, All-for-One Ecotourism, Synergistic

\section{Introduction}

Liu Ruhai proposed in 2000 that China Western Development should guide by the Two Wings strategy which prioritizes two urban clusters as vanguards. One of the two wings is Chengdu-Chongqing Urban Belt extending from the municipality Chongqing to Chengdu, with Chongqing as the core. From which Chengdu-Chongqing Urban Cluster was taking shape. On February 2004, key leaders of party and government in Chongqing led a high-standard delegation of more than a hundred people to Chengdu and met with the delegation of party and government in Sichuan province. On the meeting, Framework Agreement on Strengthening Sichuan-Chongqing Economic and Social Cooperation for the 
Development of the Upper Yangtze River Economic Zone was signed, putting further forward establishment of the dual-core Sichuan-Chongqing Urban Cluster. On April 12, 2016, the State Council issued Reply on the Development Planning of Chengdu-Chongqing Urban Cluster (State Letter [2016] No. 68), and approved Chengdu-Chongqing Urban Cluster Development Plan. Thus Chengdu-Chongqing Urban Cluster was officially approved and authorized by the state.

Many scholars have conducted in-depth studies on Chengdu-Chongqing Urban Cluster. Some scholars mainly focus on transportation layout and optimization, such as Tang Yi (2018), Li Geng (2018). While others, for example, Xia Tianci (2018) and Hu Dongyang (2018) pay attention to space structure and functional division. But the study on tourism is relatively rare, especially on all-for-one ecotourism. In 2014, President Xi Jinping brought up the idea that lucid water and lush mountains are invaluable assets so we need to speed up ecological conservation. National Tourism Administration of National Development and Reform Commission issued National Ecotourism Development Plan (2016-2025) on August 2016. National Tourism Administration announced the year 2018 as "Beautiful China-The Year of All-for-one Tourism" on January 12,2018 . All-for-one tourism will be an inevitable choice for social development. To increase the amount of studies on all-for-one ecotourism development of Chengdu-Chongqing Urban Cluster is not only to adapt to the development of China's society and economy, but also to put into practice Chengdu-Chongqing Urban Cluster Development Plan. Furthermore, it will strengthen the comprehensive competitiveness of Chengdu-Chongqing Urban Cluster.

\section{Regional Survey of Ecotourism in Chengdu-Chongqing Urban Cluster}

Chengdu-Chongqing Urban Cluster covers an area of 185 thousand square kilometers, including 15 cities of Sichuan province: Chengdu, Zigong, Luzhou, Deyang, Mianyang (exclude Beichuan and Pingwu counties), Suining, Neijiang, Leshan, Nanchong, Meishan, Yibin, Guang'an, Dazhou (exclude Wanyuan county), Ya'an (exclude Tianquan and Baoxing counties), Ziyang; and 27 districts (counties) of Chongqing: Yuzhong, Wanzhou, Qianjiang, Fulin, Dadukou, Jiangbei, Shapingba, Jiulongpo, Nan'an, Beibei, Qijiang, Dazu, Yubei, Banan, Changshou, Jiangjin, Hechuan, Yongchuan, Nanchuan, Tongnan, Tongliang, Rongchang, Bishan, Liangping, Fengdu, Dianjiang, Zhongxian, as well as some areas of Kaixian and Yunyang counties. With rich ecotourism resources, the cluster enjoys a sound trend of ecotourism development.

\subsection{Affluent Flora and Fauna Resources}

The cluster is home to 27 species of rare animals under prioritized national protection such as giant panda and red panda; and 18 species of rare plants under prioritized national protection such as spinulose tree fern and gingko biloba, 
forming 18 ecotourism scenic spots of biology and landscape like Huangjing Virgin Forest and Qingxi Valley \& Huatianjiudi Scenic Spot.

\subsection{Various Water Bodies and Rich Water Resources}

Big rivers such as Minjiang River, Jialing River and Yangtze River gather here, with lakes scattered around such as magnificent Qinglong Waterfall and beautiful Xiaonanhai Lake. The cluster boasts hot-spring tourism attractions with unique characteristics such as Feiyun Hot Spring in Kaijiang county, Daying Dead Sea and Tongjing Hot Spring; and ecotourism scenic spots of water area landscape such as Shixianghu Lake, Hongkou Natural Reserve, Fenghuanghu Scenic Resort, Dead Sea of China, Shuitianhuayue Scenic Spot in Meishan county, Bashan Grand Canyon, Xiaolanhai Scenic Spot, Beiquan Scenic Resort, Weixinghu Lake and Changshouhu Lake of Chongqing.

\subsection{Many Types of Landform}

There are mountains, gorges, karst caves, karst landform and Danxia landform, forming ecotourism scenic spots of land surface such as Xiling Snow Mountain in Chengdu, Emei Mountain, Mengding Mountain, Stone Sea in Xinwen county of Yibin city, Xishan Mountain in Nanchong, Fobao Secnic Spot in Hejiang county of Luzhou city, Foye Karst Cave in Jiangyou, Jinyun Mountain of Chongqing, Great Rift Valley of Wuling Mountain in Fuling county.

\subsection{Time-Honored History and Distinctive Cultural Ecotourism}

The cluster is the hometown of many great men living in ancient or modern times. There is Du Fu Thatched Cottage in Chengdu, Li Bai Memorial Hall, Yuan Zhen Memorial Hall, Sansu Temple Museum and many former residences of historic figures like Deng Xiaoping, Zhu De, Zhang Aiping, Liu Bocheng, and Yang Angong. Religious cultures prosper here. There are Qingcheng Mountain of Taoist, Emai Mountain of Buddhist, Zhenfo Mountain of Buddhist, Taoist and Confucianist in one. The cluster is an area where the culture of three kingdoms flourished. There is Wuhou Temple in Chengdu, Langzhong Ancient Town, Xishan Mountain Scenic Spot in Nanchong. It is a key place where red culture developed. There are Memorial Hall of Defense War in Wanyuan, Huaying Mountain Scenic Spot (the place where the story of an old lady with two guns occurred), Zhazi Cave Tourism Spot and Baigongguan Mansion Tourism Spot. Ancient Shu civilization lied here. There are Jinsha Ruins Museum in Chengdu and Sanxingdui Ruins Museum in Guanghan. Jiezi Ancient Town, Pingle Ancient Town, Lizhuang Ancient Town, Ciqikou Ancient Town and Longtan Ancient Town are also located within the cluster.

As what has been seen, various ecotourism products have taken shape in Chengdu-Chongqing Urban Cluster, with 4 world cultural and natural heritages [1] and 9 5A-class tourist attractions [2] (see Table 1, Table 2 for details). The development trend of ecotourism resources remains sound. 
Table 1. Scenic Spots in Chengdu-Chongqing Urban Cluster entered in World Heritage List.

\begin{tabular}{cccc}
\hline Number & Scenic Spots & Time of Entry & Affiliated Area \\
\hline 1 & Emei-Leshan Mountains Scenic Spot in Sichuan & 1996.12 & Sichuan province \\
2 & Qingcheng Mountain and Dujiangyan Irrigation & 2000.11 & Sichuan province \\
3 & System Scenic Spot in Sichuan & & Sichuan province \\
4 & Giant Panda Habitat in Sichuan & 2006.7 & Chongqing city \\
\hline
\end{tabular}

Table 2. 5A-Class Scenic Spots of Chengdu-Chongqing Urban Cluster.

\begin{tabular}{cccc}
\hline Number & Scenic Spots & Time of Entry & Affiliated Area \\
\hline 1 & Qingcheng Mountain-Dujiangyan Irrigation System & 2007 & Dujiangyan \\
2 & Scenic Spot in Chengdu & 2007 & Leshan \\
3 & Leshan-Emei Mountains Scenic Spot & 2011 & Emeishan \\
4 & Leshan Giant Buddha Scenic Spot & 2013 & Guang'an \\
5 & Deng Xiaoping's Hometown in Guang'an & 2013 & Nanchong \\
6 & Zhu De’s Hometown-Linglang Mountain Scenic Spot & 2016 & Nanchong \\
7 & Dazu Rock Carvings in Chongqing & 2007 & Chongqing city \\
8 & Simian Mountain Scenic Spot in Jiangin & 2015 & Chongqing city \\
9 & Longgang Scenic Spot in Yunyang of Chongqing & 2017 & Chongqing city \\
\hline
\end{tabular}

\section{Problems in Development of Ecotourism of Chengdu-Chongqing Urban Cluster}

\subsection{Severe Environmental Destruction}

National Ecotourism Development Plan (2016-2025) points out "ecotourism is based on the concept of sustainable development; guided by the principle of harmony between man and nature; and on the premise of ecological environment protection. It is a way of tourism to conduct ecological experience, ecological cognition and ecological education and acquire happiness in both body and mind, by appreciating natural ecological environment and its co-existing cultural ecology". But at present, the actual situation of Chengdu-Chongqing urban cluster is that the planning of most tourist attractions has lagged behind or not developed in accordance with the established planning ideas and plans. It appears that the concept of sustainable development has not been injected into the development process of tourist attractions, and the criterion of realizing the harmony between human beings and nature has not been adhered to. Without the premise of protecting the ecological environment, the ecosystem was destroyed by disorderly construction and sewage discharge in the tourist area. For example, the Shenlong Gorge scenic spot in Nanchuan, Chongqing, was revoked by the National Tourism Administration (now the Ministry of Culture and Tourism) because of the problems of low prices, poor sanitation and poor ser- 
vice quality. Due to similar problems, Chengdu China Loquat Expo Park was revoked 3A scenic spot qualification, Mianyang Fenghuangshan scenic spot, Guangan Huaying Xianhedong scenic spot was revoked 2A scenic spot qualification. Besides, some tourists lacking of environmental protection awareness drop litter carelessly, resulting in severe destruction of ecotourism resources.

\subsection{Backward Ecotourism Infrastructure}

Infrastructure, the first stage of the ecotourism development, presents the largest "bottleneck" of ecotourism development of Chengdu-Chongqing Urban Cluster. In the aspect of transportation, majority scenic spots of the cluster are located far away from the city center. Taking the 4A-class tourist attraction Bashan Grand Canyon as an example, according to the app Baidu Map, it stands 140 kilometers away from the center of Dazhou city and spends over 3 hours by self-driving. Thus, it is not convenient to get there. Transportation network between and within cities and districts remains poor, making it hard to conduct united development. In the aspect of service facilities, bathrooms in these spots usually fail to meet tourists' need; facilities of shopping, entertainment, restaurants and hotels are under no unified governance, leading to unpleasant experience of tourists.

\subsection{Shortage of Ecotourism Talents}

Ecotourism industry produces small destruction to natural resources while creates high economic value. A team of highly-competent managerial staffs is needed. Located at western part of China, the cluster is relatively lagged behind in education; short of local ecotourism managerial talents; lacking of attractiveness to the talents from other places due to not-so-well payment, resulting in a management team consisting of incompetent staffs; limited by low management efficiency; lacking scientific decision making; and with low service efficiency [3].

\subsection{Insufficient Synergistic Development}

Chongqing and Sichuan signed a tourism cooperative agreement in 2007 in order to promote complementary of tourism advantages among scenic spots involved for a healthy development of the tourism industry. Ziyang and Chongqing signed a cooperative agreement to jointly apply the entry into the world heritage list and open up a two-day route crossing these two places. Sichuan Tourism Administration and Chongqing Tourism Administration officially signed "Intercity Tourism-Memorandum of Strategic Cooperation in Chengdu-Chongqing Tourism Economic Cooperation Demonstration Zone" in 2011. Guang'an officially joined in West-Chongqing Tourism Alliance in 2015 and developed tourism together with 11 counties or districts in western Chongqing. 15 tourism departments in Chengdu, Suining, Panzhihua, Hechuan, Tongnan and so on jointly signed a cooperative agreement to build up Suining-Chongqing tourism corridor in 2016. The signing and implementation of these agreements laid part of 
foundation for the synergistic development of all-for-one ecotourism of Chengdu-Chongqing Urban Cluster, but none of them brought up a systematic planning and overall arrangement for it, resulting in that the cluster failed to produce full economic and social effects.

\section{Solutions to Problems in Synergistic Development of All-For-One Ecotourism of Chengdu-Chongqing Urban Cluster}

With abundant tourism resources, Chengdu-Chongqing Urban Cluster has all along been integrated since ancient times. But an array of problems, such as similarity of resources, redundant construction and illicit competition, emerged in those scenic spots. As the national government attaches great importance on and seeks to boost the cluster, a synergistic development of all-for-one ecotourism represents not only an irresistible and welcome trend, but also a need for market development. Concerted efforts from government, tourism businesses and tourists are needed for the synergistic development of all-for-one ecotourism of Chengdu-Chongqing Urban Cluster.

\subsection{Government}

The 13th Five-Year Plan of Tourism Development issued by National Tourism Administration in 2017 proposes to strengthen five tourism urban clusters. Of which, the positioning of Chengdu-Chongqing Tourism Urban Cluster is to give full play to roles of core cities in the upper reaches of Yangtze River; and, based on unique ecology and culture of Sichuan and Chongqing, to build up a high-quality international tourism cluster with both natural and cultural heritages and the competence to influence tourism of western China. Therefore, governments of Sichuan and Chongqing have to fully explore ecological and cultural characteristics of the cluster in the process of planning as so to delivery feasible plan in all aspects for ecotourism development of the cluster. At the same time, given that ecotourism resources of the cluster are mostly located in economically backward areas, investment in the development of ecotourism resources in these areas should be increased, especially in infrastructure such as highways.

\subsection{Tourism Businesses}

Tourism businesses play a vital role in ecotourism planning and tourism management. In order to boost all-for-one ecotourism of Chengdu-Chongqing Urban Cluster, it is necessary to improve tourism businesses' awareness to protect ecological environment and cooperate, publicize the idea of all-for-one ecotourism, explore ecotourism features of the cluster, develop and design distinctive ecotourism products, and open up high-quality ecotourism routes on the premise that environmental carrying capacity is fully considered so as to activate all-for-one ecotourism of the cluster. In addition, talent is a key factor to the 
quality of ecotourism planning and management, but also a shortcoming in the ecotourism development of the cluster. We should focus on cultivating and introducing a group of managerial talents professional in both tourism and ecology. Meanwhile, it is a need to strengthen ecotourism expertise training for existing tourism talents and residents in scenic spots.

\subsection{Tourists}

Tourists are direct beneficiaries of ecotourism. Tourists should be demanded to protect ecological environment and tourism resources without reminder. To achieve this, it is a must to enhance tourists' awareness of ecological protection through a large-scale publicity. Besides, relevant legal systems should be established to give appropriate punishments to those who damage ecotourism resources [4].

\section{Conclusion}

Chengdu-Chongqing Urban Cluster enjoys affluent ecotourism resources, but the socioeconomic effect of ecotourism of the clusters not significant due to backward infrastructure. A synergistic development of all-for-one ecotourism will serve as a driver for the cluster to create more ecotourism resources, produce various ecotourism products, optimize the layout of ecotourism resources, and pursue sustainable development that features in a livable and picturesque environment, providing an ecological safeguard for socioeconomic development of Chengdu-Chongqing Urban Cluster and making contributions to build a beautiful China.

\section{Acknowledgements}

This work was sponsored by Sichuan Provincial Social Sciences Research High-level Research Team: Sichuan Old Revolutionary Base Areas' Ecological Civilization Construction Research Team (Project No.: 18SCTD01), the Open Research Subject of Tourism Development Research Center of Sichuan (SCTRC) (Project No: LYC18-47), Sichuan Landscape and Recreation Research Center (Project No.:JGYQ2018036), Development and Research Center of Sichuan Old Revolutionary Base (Project No: SLQ2017C-09).

\section{Conflicts of Interest}

The authors declare no conflicts of interest regarding the publication of this paper.

\section{References}

[1] 53 World Heritage Sites in China. Zhengyi Art. http://www.zyzw.com/twzs010.htm

[2] Ranking of 5A Tourist Scenic Spots in China and List of the Latest National 5A Scenic Spots in 2018. Cloud Tour. 
http://www.lvyougl.com/fengjing/330053.html

[3] Fu, Y.Y. and Liu, Q. (2002) Current Situation and Development Suggestions of Ecotourism in Western China. Geography and Territorial Studies, No. 5, 103-106.

[4] Peng, W.Y. and Peng, X.J. (2008) The Current Development Situation of Eco-Tourism Based on Chongqing. Industrial Economy, No. 2, 259-260. 